\title{
EL DERECHO A LA AUTONOMÍA DE LOS PUEBLOS INDÍGENAS DE MÉXICO: Una aproximación desde los Derechos Humanos
}

\author{
The right to autonomy of Indigenous Peoples of Mexico: \\ A human rights approach
}

\begin{abstract}
"Los pueblos indígenas, en ejercicio de su derecho de libre determinación, tienen derecho a la autonomía o al autogobierno en las cuestiones relacionadas con sus asuntos internos y locales, así como a disponer de los medios para financiar sus funciones autónomas". Artículo 4 de la Declaración de las Naciones Unidas sobre los Derechos de los Peblos Indígenas.
\end{abstract}

José Jesús Soriano Flores

Sumario:

I. Consideraciones Iniciales. II. Discriminación y Pueblos Indígenas: la vulneración de Derechos. III. Algunas referencias relativas a los Derechos Humanos Colectivos de los Pueblos Indígenas. IV. Una aproximación al Derecho a la Autonomía de los Pueblos Indígenas. V. Apuntes Finales.VI. Bibliografía.

Resumen: El artículo establece el planteamiento del problema respecto a la vigencia del Derecho Humano a la Autonomía de los Pueblos Indígenas de México, partiendo de elementos normativos como la reciente Reforma Constitucional en materia de Derechos Humanos de 2011, la Ley Estatal en la materia, y por supuesto el Derecho Internacional de los Derechos Humanos.

Palabras Clave: Pueblos Indígenas, Autonomía, Derechos Humanos, Principio Pro Persona.

Abstract: The article sets out the problem regarding the validity of the Human Right to Autonomy of Indigenous Peoples of Mexico, based on regulatory elements as the recent constitutional reform on Human Rights, 2011, State law on the subject, and of course the International Law of Human Rights.

Keywords: Indigenous, Autonomy, Human Rights, Pro Persona Principle.

\section{CONSIDERACIONES INICIALES}

Escribir desde los Derechos Humanos, es decir, asumir una postura coincidente con la idea de dignidad humana, implica asumir también compromisos inaplazables. Es por ello, que

* Profesor Investigador de Tiempo Completo del Departamento de Derecho de la División de Derecho, Política y Gobierno de la Universidad de Guanajuato; y Secretario de la Red Estatal de Profesoras y Profesores en Derechos Humanos. Correo electrónico: jesussorianomx@yahoo.com.mx

Maestro en Derecho Constitucional por la Universidad Iberoamericana y el Instituto de Investigaciones Jurídicas de la UNAM, Licenciado en Derecho por la Universidad de Guanajuato y alumno del Programa de Doctorado Interinstitucional en Derecho de las Universidades Públicas de la Región Centro Occidente de ANUIES. Miembro del Cuerpo Académico: Ciencia Penal, Estado de Derecho y Derechos Humanos. 
trazar líneas sobre estos temas, es una extraordinaria oportunidad que permite explicar a las y los lectores, las múltiples necesidades que en materia de estas prerrogativas fundamentales existen, las cuales se multiplican en el caso de los denominados "grupos en situación de vulnerabilidad", entre los que se encuentran los pueblos indígenas.

La problemática que abordaremos, no es privativa del caso mexicano, en la orbe completa, que tiene aproximadamente trescientos millones de indígenas, ${ }^{1}$ encontramos elementos semejantes que los caracterizan. No obstante este paréntesis, en el desarrollo del texto me referiré particularmente ideas relativas a nuestro país.

La búsqueda de significados a los problemas que padecen los pueblos indígenas de México, necesariamente nos remite al año de 1492, tiempo en el que los europeos, al haber creído que encontraban las indias, denominaron precisamente "indios", a los habitantes nativos del continente que habitamos. Y el problema es que esta denominación, desde esa época, integró múltiples elementos que consideraban al indígena como una persona inferior, denominación, que lamentablemente conserva en la actualidad algunos de esos elementos, es decir, el término indio o indígena, nace con una carga discriminatoria que sigue reproduciendo sus significados.

Como lo señala el Dr. Jorge González Galván, cuya obra jurídica recomiendo ampliamente si se quiere adoptar un modelo normativo respetuoso de los derechos humanos y en la ruta del pluralismo jurídico, "en los casi cinco siglos recientes, los pueblos indios de México han coexistido en una sociedad que los excluye; los procesos colonial y nacional no reflejaron en sus naciones ni en la realidad, el respeto a las diferencias culturales".

Múltiples son las acciones que se han emprendido con el fin de eliminar a los pueblos indígenas, ${ }^{3}$ sin embargo, estos pueblos existen, y sobreviven en las condiciones socioeconómicas más precarias, pues el mapa indígena, encaja casi perfectamente en el mapa de la pobreza extrema de nuestro territorio, situación que no únicamente encuentra su raíz en factores económicos, sino también sociales, políticos, históricos y jurídicos, siendo este último factor, el tema central del texto.

Hablar y pensar en el lenguaje de los Derechos Humanos desde una perspectiva estrictamente jurídica implica traer a la reflexión conceptos novedosos, y más aun tratándose de las prerrogativas fundamentales de uno de los grupos más desprotegidos institucionalmente a lo largo de nuestra historia: los Pueblos Indígenas; y la novedad de referencia, fundamentalmente tiene que ver con las siguientes dos recientes apariciones legislativas que deberán interpretarse de conformidad con el Derecho Internacional de los Derechos Humanos:

La importante Reforma Constitucional en materia de Derechos Humanos de Junio del presente año (2011), que entre otras cosas modificó la denominación del primer capítulo de la Constitución Federal, antes llamado "De las Garantías Individuales", y ahora "De los Derechos Humanos y sus Garantías", con implicaciones trascendentes que muchos, desde años atrás, veníamos señalando como pertinentes.

\footnotetext{
${ }^{1}$ Según datos de la Organización de las Naciones Unidas (ONU)

2 González Galván, Jorge Alberto, Derecho Indígena, $1^{a}$ ed., UNAM, Instituto de Investigaciones JurídicasMcGraw-hill, México, 1997, p. 32.

3 En ocasiones mediante Políticas Públicas de Integración (“política indigenista”). 
La Ley para la Protección de los Pueblos y Comunidades Indígenas en el Estado de Guanajuato. ${ }^{4}$

En este tenor, por medio de este artículo y con más herramientas jurídicas, reafirmamos una serie de ideas teóricas relativas precisamente a la vigencia de los Derechos Humanos de los Pueblos Indígenas, ${ }^{5}$ vigencia absolutamente necesaria para vivir en Estados verdaderamente democráticos.

Y ahora que nos referimos a democracia, entendiéndola en el marco de una construcción histórica de ese concepto, podemos identificarla en una de sus acepciones, y en palabras del Profesor de la Universidad de Yale, Robert Alan Dahl, como "un mecanismo mediante el cual los diversos grupos que se encuentran en una comunidad dirimen sus discrepancias y se ponen provisionalmente de acuerdo sobre una definición de las reglas común para todos", 6 acepción idónea para entender la tesis central de estas líneas y que se traduce en la aproximación al Derecho a la Autonomía de los Pueblos Indígenas, como un elemento primordial para la vigencia del Estado Democrático.

Efectivamente son evidentes diversos avances en la construcción de nuestro Estado Democrático, no obstante ese mecanismo del que hablaba Dahl cuando se definía a la democracia, en la relación pluricultural de nuestro Estado Mexicano, aun no logra encontrar las mejores vías hacia la vigencia de los Derechos Humanos colectivos de los pueblos indígenas, por ello es importante reafirmar los contenidos esenciales de algunas de estas prerrogativas; que en esta ocasión centraremos en el derecho a la autonomía que se inscribe dentro de los denominados "derechos colectivos".

Los obstáculos en la vigencia de los Derechos Indígenas, están íntimamente relacionados con el problema de la Discriminación, en el siguiente apartado, antes de referirnos a los derechos colectivos y a la autonomía, revisaremos algunas notas al respecto.

\section{DISCRIMINACIÓN Y PUEBLOS INDÍGENAS: LA VULNERACIÓN DE DERECHOS}

Despertemos, seamos el metal derretido, lo que quiera la sed, la tierra trabajada, lo que quieran las piedras, la sencillez del huerto, lo que pidan las llamas, en fin-al fin-la piel abierta en surco.

Juan Bañuelos

La indiferencia y la insensibilidad, cuando hablamos de los pueblos indígenas, se hacen presentes en nuestra sociedad de manera espontánea, y es que la marginación de los mismos se manifiesta como algo normal, o peor aún, lo que algunos piensan: como algo que ellos quieren, que ellos mismos decidieron. Dentro de esta idea, es importante recordar algunos de los resultados de la Segunda Encuesta Nacional sobre Discriminación en México de 2010

\footnotetext{
${ }^{4}$ Publicada en el Periódico Oficial del Gobierno del Estado número 56, segunda parte, de fecha 8 de Abril de 2011, y con un rezago de casi 10 años, pues recordemos que la reforma a la Constitución Federal del mes de Agosto de 2001, obligaba a las legislaturas estatales a realizar adecuaciones en la materia.

${ }^{5}$ Se recomienda consultar el Folleto Derechos Humanos de los Pueblos y Personas Indígenas: 10 Preguntas Básicas, editado por la Procuraduría de los Derechos Humanos del Estado de Guanajuato en Marzo de 2007, editado por Manuel Vidaurri Aréchiga, Fátima Rostro Hernández y Jesús Soriano Flores.

6 Bellamy Richard, "Norberto Bobbio: Estado de Derecho y Democracia”, en DOXA, Cuadernos de Filosofía del Derecho, Núm. 28, Universidad de Alicante, España, 2005, p. 79.
} 
realizada por el Consejo Nacional para prevenir la Discriminación (CONAPRED), ${ }^{7}$ en la que las minorías étnicas opinan que precisamente la discriminación es su problema más grave, y encontramos datos como los siguientes:

- Casi cuatro de cada diez miembros de un grupo étnico consideran que no tienen las mismas oportunidades que los demás para conseguir trabajo.

- Tres de cada diez consideran que no tienen las mismas oportunidades para recibir apoyos del gobierno.

- Uno de cada cuatro dijo no tener las mismas oportunidades para tener acceso a servicios de salud o educación, etc.

Es importante manifestar, que los problemas de los pueblos indios no solamente tendrán solución cuando estos adquieran mejores condiciones económicas, sino que solamente, esta sería una de las manifestaciones si la justicia social tuviera eficacia para estas comunidades. El problema esencial, creemos, está salpicado primordialmente de factores culturales (entre los que se encuentra el jurídico), y no precisamente económicos.

Es indispensable que tengamos perfectamente claro que nuestra nación constituye una comunidad política, pero no una comunidad cultural (sino pluricultural). Debemos decir esto muy fuerte; este es un problema nacional, y es tiempo ya, de que las dificultades en la vigencia de los derechos de las personas que compartimos el territorio mexicano, sean una preocupación general, sobre todo porque el tema que nos ocupa, se extiende a más de cinco siglos y se presenta como una de las acciones más discriminatorias de todos los tiempos.

El comienzo del siglo pasado con una Revolución, fue sin duda uno de los momentos más importantes en los que los pueblos indios creyeron que por fin encontrarían la solución paulatina a sus problemas, al respetarse su forma de ser y de actuar, sin embargo, este respeto no se hizo presente. Los regímenes que surgieron de la Revolución de 1910, consideraron que no se podía concebir un desarrollo integral del país, con una variedad cultural, por lo que las políticas públicas debían propiciar el establecimiento de una sola lengua, una sola religión, un solo derecho, en resumen, una unidad cultural, violentando de esta manera los derechos humanos de los pueblos indígenas, mediante la integración.

Actualmente, el Derecho a la No discriminación, por fortuna forma parte del artículo 1 de la Constitución Federal desde el año 2001 y su justiciabilidad y exigibilidad, cada vez tienen más y mejores vías para hacerlo efectivo desde el punto de vista jurídico, que sólo constituye una de las aristas del problema de referencia.

\footnotetext{
${ }^{7}$ A través de la Enadis 2010, se actualiza el panorama de discriminación que persiste en México y se profundiza el conocimiento sobre quién o quiénes discriminan, en qué ámbitos de la vida se presenta este problema con mayor frecuencia y los factores socioculturales que se le relacionan. Se brindan elementos para conocer las percepciones de la discriminación entre la población en general y desde los distintos grupos que viven situaciones que les hacen vulnerables a la discriminación.
} 
III. ALGUNAS REFERENCIAS RELATIVAS A LOS DERECHOS HUMANOS COLECTIVOS DE LOS PUEBLOS INDÍGENAS

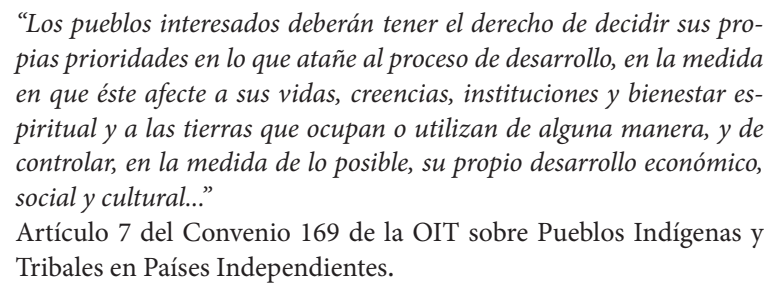

Los seres humanos, tenemos derechos individuales y colectivos, así por ejemplo, tenemos el derecho individual a la libertad de tránsito o expresión; pero también derecho a la libre determinación, ésta última prerrogativa que solamente podemos concretar como pueblos, es decir, "colectivamente". Los derechos humanos específicos de los pueblos indígenas o indios, ${ }^{8}$ o los denominados derechos étnicos, son en consecuencia derechos colectivos, esto es, derechos reclamados por una colectividad, como lo son los indígenas o los pueblos indios, en contraposición a los derechos individuales (en donde el sujeto de derecho es el individuo). 9

Para reforzar esta idea, y tener una noción más clara del significado de los derechos colectivos, recordaremos algunos de los argumentos de Rodolfo Stavehagen, destacado especialista en la materia. ${ }^{10}$

En primer lugar, es preciso reconocer que ciertos derechos humanos individuales, solamente pueden ser ejercidos plenamente en forma colectiva.

En segundo lugar, siendo la naturaleza del ser humano eminentemente social, las principales actividades alrededor de las cuales se ha constituido el debate sobre los derechos humanos, se realizan en grupos y colectividades con personalidad propia. En consecuencia el ejercicio de numerosos derechos humanos, solamente puede realizarse en el marco de estas colectividades, que para ello deberán ser reconocidas y respetadas como tales por el Estado y la sociedad en su conjunto.

Por lo tanto, la conclusión provisional y normativa que emana de estos razonamientos de Stavenhagen es muy precisa: "los derechos grupales o colectivos deberán ser considerados como derechos humanos en la medida en que su reconocimiento y ejercicio promueva a su vez los derechos individuales de sus miembros", ${ }_{11}$ tal y como ocurre con la "autonomía", presupuesto fundamental para ejercer otros derechos.

\footnotetext{
${ }^{8}$ Recordemos que ambas expresiones son válidas de acuerdo a los propios indígenas.

9 Ordóñez Mazariegos, Carlos Salvador, “Derechos Humanos de los Pueblos Indios”, en Etnicidad y Derecho: un diálogo postergado entre los científicos sociales, $1^{\mathrm{a}}$ ed., UNAM, México, 1996, p. 215-216.

${ }^{10}$ Idem.

${ }^{11}$ López y Rivas, Gilberto, Nación y Pueblos Indios en el Neoliberalismo, 2a . ed., UIA, México 1996, p. 31.
} 


\section{UNA APROXIMACIÓN AL DERECHO A LA AUTONOMÍA DE LOS PUEBLOS INDÍGENAS}

"El Estado reconoce el derecho de los pueblos y las comunidades indígenas a ejercer su autonomía".

Artículo 15 de la Ley para la Protección de los Pueblos y Comunidades Indígenas en el Estado de Guanajuato.

Como lo hemos planteado en otros estudios, ${ }^{12}$ la Tierra y Territorios, la Cultura y la Autonomía, son algunos de los derechos colectivos de los pueblos indígenas, pero en esta ocasión únicamente ahondaremos en el derecho a la autonomía, que constituye una de las demandas más importantes que encabeza el movimiento indio.

Jurídicamente podemos decir que autonomía consiste en "la facultad que las organizaciones políticas tienen de darse a sí mismas sus leyes, y de actuar de acuerdo con ellas". ${ }^{13} \mathrm{o}$ bien atendiendo a la nueva ley en Guanajuato (2011), ${ }_{14}^{14}$ que identifica a la autonomía como "la expresión de la libre determinación de los pueblos y las comunidades indígenas como partes integrantes del Estado, de conformidad con el orden jurídico vigente, para adoptar por sí mismos decisiones e instituir prácticas propias relacionadas con su manera de ver e interpretar las cosas, con relación a su territorio, recursos naturales, organización sociopolítica, económica, de administración de justicia, educación, lenguaje, salud y cultura, que no contravengan la unidad nacional". ${ }^{15}$

Los derechos colectivos son tan importantes, que sin ellos, los derechos individuales no tienen un ejercicio completo, recordemos que una de las características más importantes de los Derechos Humanos es precisamente su "integralidad", que significa entre otras cosas, que estos derechos constituyen un compendio único que no se separa, a menos que se tengan finalidades didácticas, en éste último caso, es precisamente cuando se habla de clasificaciones de los Derechos (Civiles, Políticos, Sociales, Culturales, Ambientales etc.), que dejaron atrás la idea de las generaciones de los mismos.

Nuevamente haciendo primeramente referencia a la persona individual, podemos decir que "los derechos humanos individuales, comprenden el derecho a la autonomía de la persona”, y como atinadamente lo apunta Luis Villoro, los individuos no son individuos sin atributos, incluyen la conciencia de la propia identidad como miembros de una colectividad, y ésta no puede darse más que en un contexto cultural. Autonomía de la persona es la capacidad de elegir conforme a sus propios fines y valores, y de ejercitar esa elección. Y los fines y valores están determinados por el marco de una cultura determinada. Las creencias básicas que determinan las razones válidas, los fines elegibles y los valores realizables pueden variar de una cultura a otra. Por lo tanto, el reconocimiento de la autonomía de las personas implica el de las comunidades culturales a las que pertenecen.

\footnotetext{
${ }^{12}$ Véase: Soriano Flores, José Jesús, "Reflexiones acerca del tratamiento constitucional de los Derechos Humanos de los Pueblos Indígenas”, en Libro Homenaje al Profesor Dr. Héctor Fix Zamudio, Cuarezma Terán, Sergio (Coord.), $1^{\mathrm{a}}$ ed., Corte Suprema de Justicia de Nicaragua y otras, Nicaragua, 2010, p. $287-307$.

${ }^{13}$ García Máynez, Eduardo, Introducción al Estudio del Derecho, 48ª ed., Porrúa, México, 1996, p.104.

${ }^{14}$ Artículo 6, Fracción I de la Ley para la protección de los pueblos y comunidades indígenas en el estado de Guanajuato; publicada en el Periódico Oficial del Gobierno del Estado de Guanajuato, número 56, segunda parte, de fecha 8 de Abril de 2011.

${ }^{15}$ A pesar del avance en el reconocimiento legislativo del derecho a la autonomía en Guanajuato, es evidente el rezago conceptual, al hacer referencia a ese posible atentado contra la unidad nacional, pues como lo veremos en líneas posteriores, es un mito que parte de la confusión de conceptos.
} 
El derecho de los pueblos sólo puede contarse entre los derechos humanos, en la medida en la que el pueblo sea una condición para la autonomía de las personas; en esa medida sólo puede referirse a la comunidad cultural en cuyo marco se da cualquier elección autónoma; en esa medida también el derecho de los pueblos, no contradice los derechos del individuo, sino, por el contrario, los refuerza. Y en este orden de ideas, Luis Villoro se pregunta, ¿qué debe comprender un derecho de los pueblos, para ser considerado entre los derechos humanos fundamentales?, concluyendo que debe incluir, desde luego los derechos individuales, y que además cuando la asociación política se establece entre sujetos pertenecientes a una misma cultura, comprende el respeto a la autonomía de esa comunidad cultural. Así tenemos a la autonomía como un derecho humano colectivo, que está estrechamente ligado a otros derechos como a la cultura y territorio. ${ }^{16}$

Uno de los lugares comunes en la discusión relativa a las autonomías indígenas, es la confusión entre autonomía y soberanía. ${ }^{17}$ El reconocimiento del derecho a la autonomía, no representa de ninguna manera el reconocimiento de extraterritorialidad o como se ha dicho alguna vez, el camino hacia la formación de otro Estado; autonomía significa que la reproducción de las sociedades indias se rijan económica, social y culturalmente con el ejercicio pleno de sus derechos históricos, con las intermediaciones de la sociedad nacional que corresponden a la formación de la sociedad mexicana.

El ejercicio de la autodeterminación, significa como lo ha entendido Villoro, una vinculación de los indios con el Estado en el desarrollo de la colectividad política, con lo cual, a tiempo que el bloque hegemónico reconozca a las autoridades tradicionales en sus respectivas jurisdicciones, deberá establecer espacios dentro de la actual estructura de poder (congresos estatales, presidentes municipales, etc.) en el que los indígenas cuenten con representantes de sus propias etnias. ${ }^{18}$

Continuando con este tenor de ideas, López y Rivas, señala que "las autonomías no sólo no van en contra de la unidad nacional, no sólo no constituyen problema de seguridad nacional, sino por el contrario, son la mejor forma de resolver problemas que, de continuar profundizándose, podrían llevar efectivamente al separatismo, [...] de esta manera las autonomías forman parte integral de un proyecto democrático de Estado y fortalecen la unidad nacional". ${ }^{19}$

Como se puede apreciar, el reconocimiento de estos derechos humanos colectivos, implica en buena medida la reestructuración del Estado Mexicano, evidentemente no bastan únicamente las adecuaciones legislativas, por ejemplo, por autonomía, no deberá entenderse solamente la capacidad de gestión, sino más bien "una definición política de gobernabilidad per se, a través del reconocimiento de la personalidad jurídica de las autoridades tradicionales indias y el ejercicio democrático de las poblaciones indias para elegirlas, y establecer aquellas líneas de crecimiento socioeconómicas idóneas al raciocinio indígena”."

\footnotetext{
${ }^{16}$ Villoro Luis, Los Pueblos Indios y el derecho a la autonomía, en Etnicidad y derecho..., Op. Cit., p.138.

${ }^{17}$ Como lo apunta Tena Ramírez en su libro Derecho Constitucional, mientras la soberanía consiste [...] en la autodeterminación plena, nunca dirigida por determinantes jurídicos extrínsecos a la voluntad del soberano, en cambio la autonomía presupone al mismo tiempo una zona de autodeterminación, que es lo propiamente autónomo, y un conjunto de limitaciones y determinaciones jurídicas extrínsecas, que es lo heterónomo.

${ }^{18}$ Villoro Luis, Los Pueblos Indios y el derecho a la autonomía, en Etnicidad y derecho..., Op. Cit., p.138.

${ }^{19}$ López y Rivas, Gilberto, Op. Cit., p. 21.

${ }^{20}$ Durand Alcántara, Carlos, Derechos indios en México... derechos pendientes, Universidad Autónoma de Chapingo, México, 1994, p. 179.
} 
En otras palabras, la autonomía se relaciona con el ejercicio democrático del poder, con la cual los pueblos indios pueden adecuar un modelo de crecimiento más acorde con sus propias expectativas, por ellos es que también reiteramos la importancia de estos derechos como elemento fundamental para la vigencia integral de la democracia en nuestro país.

Las autonomías son formas de ejercer el derecho a la libertad, que unánimemente es aceptado como un derecho humano, es por ello que los acuerdos de San Andrés Larráinzar ${ }^{21} a-$ puntaron es sus peticiones la autonomía para todas las comunidades que no pueden ejercer plenamente ese derecho. En su propuesta, la autonomía está estrechamente ligada con la democratización del estado-Nación.

Estamos conscientes de que como lo apunta López y Rivas, "no todos los pueblos indígenas de una nación determinada han alcanzado los niveles de compromiso político y organización, que hagan posible la conformación de procesos autonómicos de manera uniforme en todo el territorio nacional.” Y en atención a esta idea, nuestro país no es la excepción, es por eso, que una de las tareas esenciales del movimiento indígena y de sus aliados, es el desarrollo y fortalecimiento del sujeto autonómico. La autonomía, no sólo es una fórmula de gobierno, no sólo es una propuesta legislativa, es, sobre todo, una manera de actuar dentro de la nación como sujeto político independiente. ${ }^{22}$

La vigencia de este derecho, debe tener como consecuencia modificaciones sumamente complejas en las relaciones de poder y en el modo de vida política de las y los mexicanos. Desde el punto de vista legal, será imprescindible entender el Pluralismo Jurídico que impone esta nueva concepción en el reconocimiento del derecho a la autonomía.

A la luz de la razón cristalizada normativamente en Derechos Humanos, debe concretarse el sujeto autonómico, efectivizando todos los derechos humanos colectivos de los pueblos indígenas, premisa que sigue siendo un reto de nuestros días, si es que queremos vivir en democracia entendiéndola en su concepto más garantista.

\section{APUNTES FINALES}

Nuestra sociedad es profundamente discriminatoria, ${ }^{23}$ así lo expresó Don Gilberto Rincón Gallardo, ${ }^{24}$ indicando que esta clase de sociedades son fragmentadas, desiguales, proclives a la violencia y con escasos vínculos de solidaridad, y que en cambio, las sociedades donde se lucha contra la discriminación son más prósperas, más coherentes, más libres y más solidarias. Aquí se dibuja claramente uno de los retos que tenemos, y que de manera particular tiene el Derecho, haciendo uso entre otras cosas de acciones afirmativas.

\footnotetext{
${ }^{21}$ Documento que el gobierno de México y el Ejército Zapatista de Liberación Nacional firmaron en 1996 en el que se establecen acuerdos que deberían tomarse en cuenta en la Reforma Constitucional que reclamaban los pueblos indígenas a través del EZLN.

${ }^{22}$ López y Rivas Gilberto, Op. Cit., p.102

${ }^{23}$ Es común llamar despectivamente indio, a aquél que no sabe comportarse, que no tiene la aptitud de relacionarse satisfactoriamente con el resto de la sociedad, o incluso al que está sucio, al ignorante. Dice Veronique Flanet, en un libro que se llama "Viviré si Dios quiere", que "el mestizo, como en todos lados se considera superior al indígena, los inditos, los indios, la gente indígena se oye decir. Muchos van más lejos en sus calificativos: los indios son brutos, incultos, ignorantes, incapaces, etc.; algunos mestizos atribuyen la ignorancia del indígena a la falta de información y cultura. Pero son pocos los que piensan así: atribuir la torpeza del indio a la falta de información, sería en cierto modo, plantear una autocrítica y correlativamente la conciencia de la necesidad de un cambio."

${ }^{24}$ Destacado Defensor de los Derechos Humanos en México y fue el primer Presidente del Consejo Nacional para Prevenir la Discriminación (CONAPRED).
} 
Los individuos pertenecemos como parte esencial de nuestra naturaleza a colectividades, así, en el marco de esos grupos humanos se vive la vida personal y la vida colectiva, que integran la identidad del ser humano. Si dicha identidad no se respeta como en el caso de los pueblos indígenas, se vulneran derechos humanos.

La problemática indígena y específicamente la efectividad de sus derechos humanos, ha constituido y constituye un camino accidentado e inacabado, a pesar de las recientes reformas que comentamos en la parte introductoria del presente trabajo.

Por ello, como lo apuntamos, es pertinente seguir insistiendo en los contenidos de los derechos colectivos de estos pueblos, cuyo punto de partida, en buena medida se encuentra en el derecho a la autonomía, el cual debe ser entendido mediante una interpretación "pro persona" o "pro homine", acorde con el contenido del artículo 1 constitucional recientemente reformado (2011), que señala que "las normas relativas a los derechos humanos se interpretarán de conformidad con esta Constitución y con los tratados internacionales de la materia favoreciendo en todo tiempo a las personas la protección más amplia”.

Coincidimos con José Luis Caballero Ochoa, cuando indica que esta aplicación más favorable a las personas, tiene una clave de lectura, no precisamente jerárquica, pues "el sentido clásico de la jerarquización tiene por objeto evitar conflictos normativos, pero no integra ni expande, como es propio de las normas sobre Derechos Humanos", ${ }^{25}$ de esta manera, "la cláusula de interpretación conforme, es más compatible con los criterios de interpretación en Derechos Humanos, como los que se han venido desarrollando en la práctica del Estado Constitucional contemporáneo". ${ }^{26}$

En este orden de ideas, deben tenerse siempre en cuenta además de la normatividad estatal y nacional, diversos documentos internacionales que deberán consultarse ${ }^{27}$ comenzando con el Convenio número 169 sobre Pueblos Indígenas y Tribales en Países Independientes de la OIT, y por supuesto con la Declaración de las Naciones Unidas sobre los Derechos de los Pueblos Indígenas, esta última aprobada por la Asamblea General de las Naciones Unidas el 13 de septiembre de 2007.

El Derecho a la Autonomía, es pues, un elemento primordial en la vigencia de nuestro Estado Democrático, si es que coincidimos con Dahl quien definió a la Democracia como "un mecanismo mediante el cual los diversos grupos que se encuentran en una comunidad dirimen sus discrepancias y se ponen provisionalmente de acuerdo sobre una definición de las reglas común para todos"; discrepancias y acuerdos, evidentemente en nuestro país inacabados. La vigencia de los Derechos Humanos en nuestros Estados contemporáneos constituye un fiel indicador de la calidad de la Democracia, no lo perdamos nunca de vista.

Sabemos que es arduo el proceso para la reposición de la estructura del Estado Mexicano, en la que incluya a los pueblos y comunidades indígenas con todos sus derechos humanos incluyendo evidentemente la autonomía. No obstante dicho proceso es impostergable, en este sentido, es tiempo de impulsarlo desde la posición en la que nos encontremos, con vehemencia, conocimiento y sobre todo con mucho respeto.

\footnotetext{
${ }^{25}$ Caballero Ochoa, José Luis, "La cláusula de interpretación conforme y el principio pro persona (artículo 10., segundo párrafo, de la Constitución”, en Carbonell, Miguel y Salazar, Pedro, (Coordinadores), La Reforma Constitucional de Derechos Humanos: un nuevo paradigma, $1^{\mathrm{a}}$ ed., Instituto de Investigaciones Jurídicas de la UNAM, México, 2011, p. 109.

${ }^{26}$ Ibídem.

${ }^{27}$ Cfr. Vidaurri Aréchiga, Manuel, Pérez Guerra, José Manuel, (Compiladores), Derechos Humanos de los Pueblos Indígenas: Documentos Básicos, Procuraduría de los Derechos Humanos del Estado de Guanajuato, México, 2004, 200 pp.
} 
BIBLIOGRAFÍA:

BELLAMY Richard, “Norberto Bobbio: Estado de Derecho y Democracia”, en DOXA, Cuadernos de Filosofía del Derecho, Núm. 28, Universidad de Alicante, España, 2005.

CABALLERO OCHOA, José Luis, "La cláusula de interpretación conforme y el principio pro persona (artículo 10., segundo párrafo, de la Constitución”, en Carbonell, Miguel y Salazar, Pedro, (Coordinadores), La Reforma Constitucional de Derechos Humanos: un nuevo paradigma, $1^{\text {a }}$ ed., Instituto de Investigaciones Jurídicas de la UNAM, México, 2011.

DURAND ALCÁNTARA, Carlos, Derechos indios en México... derechos pendientes, Universidad Autónoma de Chapingo, México, 1994.

GARCÍA MÁYNEZ, Eduardo, Introducción al Estudio del Derecho, 48ª ed., Porrúa, México, 1996.

GONZÁLEZ GALVÁN, Jorge Alberto, Derecho Indígena, $1^{\mathrm{a}}$ ed., UNAM, Instituto de Investigaciones Jurídicas-McGraw-hill, México, 1997.

LÓPEZ y RIVAS, Gilberto, Nación y Pueblos Indios en el Neoliberalismo, 2a . ed., UIA, México 1996.

ORDÓÑEZ MAZARIEGOS, Carlos Salvador, "Derechos Humanos de los Pueblos Indios", en Etnicidad y Derecho: un diálogo postergado entre los cientificos sociales, $1^{\mathrm{a}}$ ed., UNAM, México, 1996.

Procuraduría de los Derechos Humanos del Estado de Guanajuato, Folleto Derechos Humanos de los Pueblos y Personas Indígenas: 10 Preguntas Básicas, editado por Manuel Vidaurri Aréchiga, Fátima Rostro Hernández y Jesús Soriano Flores, México, 2007.

SORIANO FLORES, José Jesús, “Reflexiones acerca del tratamiento constitucional de los Derechos Humanos de los Pueblos Indígenas", en Libro Homenaje al Profesor Dr. Héctor Fix Zamudio, Cuarezma Terán, Sergio (Coord.), $1^{\mathrm{a}}$ ed., Corte Suprema de Justicia de Nicaragua y otras, Nicaragua, 2010.

VIDAURRI ARÉCHIGA, Manuel, Pérez Guerra, José Manuel, (Compiladores), Derechos Humanos de los Pueblos Indígenas: Documentos Básicos, Procuraduría de los Derechos Humanos del Estado de Guanajuato, México, 2004.

VILLORO, Luis, Los Pueblos Indios y el derecho a la autonomía, en Etnicidad y Derecho: un diálogo postergado entre los cientificos sociales, $1^{\mathrm{a}}$ ed., UNAM, México, 1996.

LEGISLACIÓN:

Constitución Política de los Estados Unidos Mexicanos.

Convenio 169 de la OIT sobre Pueblos Indígenas y Tribales en Países Independientes.

Declaración de las Naciones Unidas sobre los Derechos de los Pueblos Indígenas. 
Ley para la protección de los pueblos y comunidades indígenas en el estado de Guanajuato; publicada en el Periódico Oficial del Gobierno del Estado de Guanajuato, número 56, segunda parte, de fecha 8 de Abril de 2011.

OTROS DOCUMENTOS:

Acuerdos de San Andrés Larráinzar, Documento que el gobierno de México y el Ejército Zapatista de Liberación Nacional firmaron en 1996 en el que se establecen acuerdos que deberían tomarse en cuenta en la Reforma Constitucional.

Consejo Nacional para Prevenir la Discriminación, Encuesta Nacional sobre Discriminación en México: Enadis 2010, en línea: http://www.conapred.org.mx/index. php? contenido $=$ pagina\&id $=424 \&$ id_opcion $=436 \& o p=436$, fecha de consulta: 30 de Junio de 2012. 
